\title{
Biophysical Analysis of Nucleic Acids
}

\section{BIOPHYSICAL METHODS}

The determination of the structure of a nucleic acid, or of any molecule, is a necessary step in beginning to understand what the molecule can do. Before one can ask how reactive it is, what ligands it can bind, or what reactions it can catalyze, one must know the structures of its reactants, its products, and its intermediates. Structures are required before functions can be understood. This unit describes the main biophysical methods that have been used to study nucleic acids, and briefly mentions some of the powerful new methods that are just appearing.

The first step in determining the structure of a nucleic acid is to establish its sequence-its primary structure. If the molecule was made by chemical or enzymatic synthesis, this may be a sufficient proof of sequence. For a natural DNA sample, the standard method is that of Sanger (e.g., see CPMB UNIT 7.4A), in which the DNA is replicated by DNA polymerase in the presence of one dideoxynucleoside triphosphate such as ddGTP. The ddGTP acts as a chain terminator, so that chain length determination by gel electrophoresis provides the sequence of G's. The reaction is repeated with each ddNTP base to determine the complete sequence. For RNA the same method is used, except with reverse transcriptase as the DNA-synthesizing enzyme.

Once a pure, single species of a nucleic acid with a known sequence has been obtained, its secondary structure (base pairing) is assessed. Measurement of its ultraviolet absorbance at $260 \mathrm{~nm}$ provides its approximate concentration. To further characterize the structure and to obtain a more accurate concentration, an absorbance melting curve is measured. Doublestranded nucleic acids have a lower absorbance per nucleotide than single-stranded nucleic acids. Stacked bases are said to be hypochromic (less absorbing) compared to unstacked bases. Thus, as a nucleic acid solution is heated, the absorbance increases as double strands melt and the bases in single strands unstack. A plot of absorbance versus temperature, or of the derivative of absorbance versus temperature, is called a melting curve. The shape of the curve - the number, widths, and positions of the maxima in the derivative curve-can reveal a great deal about the different conformations that are present in a nucleic acid, as well as the transitions that occur. Measurement of circular dichroism versus wavelength provides a more detailed picture of the conformations present in the sample. The various double-stranded conformations of nucleic acids (A, B, and Z forms; see APPENDIX $1 B$ ) have characteristic signatures in circular dichroism.

Absorbance and circular dichroism are thus used to measure concentrations and to qualitatively characterize the secondary structures of nucleic acids. They are also used to monitor covalent reactions or equilibrium binding with ligands. Reactions can also be followed by changes in the fluorescence of rare fluorescing bases in tRNA or, more commonly, of fluorescent ligands or reagents. All these spectroscopic measurements require on the order of 1 $\mathrm{mL}$ of a $0.1 \mathrm{mM}$ nucleotide solution. The small amount of material needed and the ease of measurement make ultraviolet absorption and circular dichroism the methods of choice for qualitative characterization of nucleic acids.

Atomic resolution structures- $\mathrm{X}, \mathrm{Y}, \mathrm{Z}$ coordinates for each atom — require either nuclear magnetic resonance (NMR) measurements in solution or X-ray diffraction measurements on single crystals. Both types of measurements take months of effort and at least an order of magnitude more material.

$\mathrm{X}$-ray diffraction depends on the ability to obtain single crystals that diffract well, which seems to depend largely on trial and error. Once a suitable crystal is found and the diffraction data analyzed, a complete structure of the nucleic acid is obtained. The structure includes coordinates of the molecule plus coordinates of tightly bound water molecules and ions. Very large molecules can be determined; for example, progress is being made on the structure of a ribosome.

NMR studies in solution can provide useful information short of a three-dimensional structure. For example, the detailed base pairing in an RNA can be established from a one-dimensional imino spectrum using no more material than needed for a UV absorbance spectrum. However, for atomic coordinates, two-dimensional spectra and ten times the concentration are needed. Interpretation of NMR experiments is limited by resolution of the spectra. Twodimensional spectra and isotope labeling improve the resolution, but there is a limit to the size of nucleic acids that can be analyzed. The size is increasing as stronger magnets and newer techniques become available, but a 
length of more than 100 nucleotides is very difficult at this time.

\section{X-Ray Diffraction}

The field of structural biology can be considered to have started in 1953 when Watson and Crick (Watson and Crick, 1953) interpreted the fiber X-ray diffraction data of Wilkins (Wilkins et al., 1953) and Franklin (Franklin and Gosling, 1953) on DNA. The data were consistent with a double-helical structure with complementary paired bases on the inside of the helix and phosphates on the outside. The resolution was not high, but the structural information was sufficient to revolutionize the study of biology. X-ray diffraction of crystals now gives the most accurate information about the atomic structures of nucleic acids. Positions of atoms can be determined to $0.001 \AA$ for small molecules and to $0.1 \AA$ for macromolecules. Crystals of bases, nucleosides, nucleotides, dinucleotides, and oligonucleotides revealed the preferred torsion angles around the seven bonds that characterize the conformation of a nucleotide (see APPENDIX $1 B \& 1 C$ ). The nomenclature used to describe a nucleic acid is based on the structures determined by $\mathrm{X}$-ray diffraction of crystals. B-form DNA has 2'-endo sugar puckers (pseudorotation phase angle $=162^{\circ}$ ), but a wide variation of pseudorotation angles is found. A-form RNA is more conformationally rigid, with $3^{\prime}$-endo sugar puckers (pseudorotation phase angle $=18^{\circ}$ ). The naturally occurring nucleotides have an anti conformation around the glycosidic bond in righthanded A-form and B-form nucleic acids, but left-handed Z-form nucleic acids have syn purines. Much of this work is summarized in Saenger (1984).

Recently, the emphasis of X-ray diffraction studies on DNA-containing crystals has included unusual structures, such as $\mathrm{G}$ quartets (Kang et al., 1992), DNA-drug complexes (Chen et al., 1997), DNA-protein complexes (Passner and Steitz, 1997), and oligonucleotides with chemically damaged bases pertinent to DNA mutation and repair (Lipscomb et al., 1995).

High-resolution X-ray structures of RNA molecules began with transfer RNAs in 1973 (Saenger, 1984). Several tRNA synthetasetRNA complexes, including tRNA ${ }^{\text {Ser }}$ and its synthetase (Biou et al., 1994), have since been solved. Ribozymes are novel RNA enzymes whose mechanisms are being actively pursued. Structures of hairpin ribozymes (Pley et al., 1994; Scott et al., 1995, 1996) have provided the conformation of the RNA and the location of crucial metal ions. The structure determined is that of the ground state, not the transition state, but the structure does allow plausible proposals about which torsion angles must change in order to form the transition state. The structure of the first RNA molecule large enough to have an inside and an outside has been published (160 nucleotides; Cate et al., 1996a). Here, inside indicates areas of the folded RNA that are not accessible to the solvent, as found in most proteins. The structure revealed several new types of RNA structural motifs (Tinoco and Kieft, 1997), including adenosine platforms and ribose zippers (Cate et al., 1996b). The importance of metal ionbinding sites in the folding of RNA molecules has become increasingly clear (Cate and Doudna, 1996; Cate et al., 1997; Correll et al., 1997).

$\mathrm{X}$-ray diffraction of crystals is the most powerful method to obtain accurate coordinates for molecular structures (Glusker et al., 1994). The conformation of the molecule is obtained, as are the locations of bound ions and bound water molecules, provided the resolution is high enough. Whether a nucleic acid molecule or complex will crystallize and whether the crystal will diffract to high resolution seems to be a matter of luck. One must try a wide range of crystallizing conditions, nucleic acid sequences, and chain lengths to obtain useful crystals.

Once the crystal structure of a nucleic acid is known, one must ask how it is related to solution conformations or physiological conformations. DNA tends to crystallize as A-form double helices, since the low water activity used for crystallization solvents favors the A form. However, the physiological structure of DNA is close to the $\mathrm{B}$ form. The difference between crystal and solution structures for DNA is exemplified by a crystal that showed the X-ray diffraction of both A- and B-form DNA (Doucet et al., 1989). The crystalline oligodeoxynucleotide had an A-form structure, but the soluble oligodeoxynucleotide in the solvent-filled interstices of the crystal had the fiber diffraction pattern of oriented B-form DNA. RNA tetraloops are very stable, very common hairpin loops that have been characterized by NMR in solution. When a UUCG tetraloop and a GAAA tetraloop were each crystallized, each formed a double strand with an internal loop of eight nucleotides (Holbrook et al., 1991; Baeyens et al., 1996). In crystals, the double 
helix with an internal loop apparently packed better than an intramolecular hairpin loop.

Crystal structures are static. There is local variation around the equilibrium positions of the atoms, which is quantitated by the temperature factor for each atom in the crystal. Some regions of the molecule may be so disordered that they cannot be determined in the crystal structure. Although the extent of variation and disorder can be interpreted in terms of dynamics of the molecule, the molecules are essentially frozen in the solid. However, the time dependence of a reaction, such as that catalyzed by an enzyme, can be measured in a crystal using the Laue X-ray diffraction method (Farber, 1997), which uses X rays with a wide range of wavelengths rather than the usual monochromatic $\mathrm{X}$ rays. For a chosen orientation of the crystal, the range of wavelengths produces a series of diffraction spots for the different crystal lattice spacing. The Laue diffraction method has the advantage that the full intensity of a synchrotron source can be incident on the crystal; no monochromator is needed. The synchrotron source provides short pulses of high-intensity radiation that can be used to take motion pictures of the crystal. One possible scenario is to start the reaction in the crystal with a short light pulse, then follow the changes in conformation with the synchrotron X-ray pulses.

\section{Nuclear Magnetic Resonance}

Nucleic acid structures at atomic resolution and the dynamics of these structures can be measured in solution by NMR (UNIT 7.2; Wüthrich, 1986; Roberts, 1993; James, 1995; Wemmer, 2000). Two- and three-dimensional NMR experiments provide distances between protons (nuclear Overhauser effect spectroscopy, or NOESY) and torsion angles between protons separated by three bonds (correlated spectroscopy, or COSY). Distance measurements depend on the nuclear Overhauser effect (NOE), which is proportional to the inverse sixth power of the distance between the protons. The rapid decrease of the effect with distance means that protons separated by $>5 \AA$ do not have measurable NOEs. Clearly, NMR provides local structure (protons within $5 \AA$ And three bonds). However, the local structure can also establish the global structure for compact molecules, such as many folded RNA molecules. It is less accurate for extended DNA molecules with slight bends.
NMR spectra in water provide an accurate secondary structure for a nucleic acid. The imino protons of guanine, thymine, and uracil resonate in a characteristic region between 9 and $15 \mathrm{ppm}$, but are only seen if they are exchanging slowly with water. Base-paired or otherwise protected imino protons exchange slowly; others exchange rapidly and are not measurable. The imino spectrum of a nucleic acid thus has only a few peaks and is straightforward to assign from the NOEs between adjacent base pairs. The assigned imino spectrum provides the sequence of the basepaired regions, which defines the secondary structure.

To obtain a complete three-dimensional structure, nonexchangeable protons must be assigned. This is usually straightforward for molecules of $<40$ nucleotides (Varani and Tinoco, 1991; Allain and Varani, 1997). Proton spectra and natural abundance ${ }^{13} \mathrm{C}$ spectra suffice to assign all base protons and the $1^{\prime}, 2^{\prime}, 2^{\prime \prime}$, and some $3^{\prime}$ protons. NOESY and COSY spectra of these protons can lead to atomic resolution structures. For molecules containing $>20$ nucleotides, isotope labeling is often required to allow the assignments necessary for structure determination. Uniform labeling with ${ }^{13} \mathrm{C}$ (Batey et al., 1992; Nikonowicz et al., 1992) and specific ${ }^{13} \mathrm{C}$ labeling (SantaLucia et al., 1995) allow three-dimensional and ${ }^{13} \mathrm{C}$-edited NMR experiments that can resolve overlapping two-dimensional proton spectra. Deuteration can simplify spectra by removing protons (Tolbert and Williamson, 1996); this can be done nonuniformly (Foldesi et al., 1996) to provide NMR windows for analyzing small regions of larger molecules.

Current NMR studies have focused on unusual (non-B-form) DNA structures and on all types of RNA structures. Base-base mismatches in DNA double strands (Chou et al., 1997), DNA triple strands (Wang et al., 1992; Radhakrishnan and Patel, 1994), G quartets (Williamson, 1994), and DNA-antibiotic complexes (Wemmer and Dervan, 1997) are some of the systems studied. Base-base mismatches in RNA (Wu et al., 1995; Wu and Turner, 1996), RNA G quartets (Cheong and Moore, 1992), RNA-antibiotic complexes (Fourmy et al., 1996; Recht et al., 1996), RNA-peptide complexes (Battiste et al., 1994; Puglisi et al., 1995), RNA-protein complexes (Ramos et al., 1997; Varani, 1997), and RNA-metal ion complexes (Kieft and Tinoco, 1997) have all been studied. RNA molecules that have been selected from a random pool of sequences to
Biophysical Analysis of Nucleic Acids 
specifically bind ligands have very compact, rigid structures (Fan et al., 1996; Jiang et al., 1996; Dieckmann et al., 1997; Zimmerman et al., 1997). RNA-RNA interactions as seen in pseudoknots (Shen and Tinoco, 1995), kissing hairpins (Chang and Tinoco, 1997), and tetraloop receptors (Butcher et al., 1997) characterize the motifs that fold RNA into functional molecules.

The dynamics of a nucleic acid molecule and of any part of the molecule can be assessed directly by NMR measurements. The width of each nuclear resonance peak (quantitated by $T_{2}$, the spin-spin relaxation time) depends on the motion of the nucleus. The motion is a combination of the rotation of the molecule as a whole plus any motion of the part of the molecule containing the nucleus. Measurement of $T_{2}$ plus $T_{1}$ (the spin-lattice relaxation time) plus NOEs for each nucleus can provide a detailed picture of the nanosecond dynamics of the molecule (Lipari and Szabo, 1982).

When there is exchange on a millisecond time scale between two different conformations of a molecule, the NMR spectrum can be used to quantitate the rate constants. Fast exchange produces a spectrum that is the average of the spectra in the two conformations, weighted by the amount of each conformation. Slow exchange produces a spectrum that is the weighted sum of the spectra of the two conformations. Intermediate exchange produces very broad resonances that are difficult to measure and interpret. In this context, fast means that the sum of the forward and reverse rate constants is large compared to the difference in resonance frequencies of a nucleus in the two conformations; slow means the opposite. For an NMR experiment at $600 \mathrm{MHz}$, a $0.1 \mathrm{ppm}$ difference in resonance frequencies means a frequency difference of $60 \mathrm{sec}^{-1}$. It is clear that, for rate constants in this range, some of the resonances can be in slow exchange and others in fast exchange. Thus, it is possible to learn which parts of a molecule are dynamic and undergoing changes in conformation.

Reactions that occur in the range of minutes to hours can be monitored by a series of onedimensional NMR spectra as a function of time. Thus, NMR measurements can be used to study dynamics of molecules on time scales from nanoseconds to days.

\section{Optical Spectroscopy}

Absorption, circular dichroism, and fluorescence can be used to obtain structural information about molecules, but not at atomic resolu- tion. Absorbance melting curves have long been used to study double-helix formation in nucleic acids (Gray et al., 1995; UNIT 7.3). Circular dichroism can characterize conformational changes on forming tertiary structure in RNA (Johnson and Gray, 1992). Fluorescence has been used to identify hybridization to arrays of sequences on solid supports (Pease et al., 1994). Fluorescence resonance energy transfer (FRET) has been used to measure distances between fluorophores in DNA (Murchie et al., 1989; Clegg et al., 1993) and RNA (Tuschl et al., 1994). Absorption and circular dichroism are very convenient for monitoring changes in conformation after a change, for example, in solvent, $\mathrm{pH}$, salt concentration, or temperature. The kinetics of changes in structure and conformation of nucleic acids have been extensively studied by optical spectroscopy. Temperature-jump and stopped-flow kinetics (LeCuyer and Crothers, 1994; Maglott and Glick, 1997) provide data for reactions in the millisecond range.

\section{THEORETICAL AND COMPUTATIONAL METHODS}

Theoretical analysis is indispensable in understanding the experimental methods used to obtain structure and dynamics. However, theoretical methods that can deduce structure directly from sequence are greatly hoped for and are being actively developed. Useful reviews of the computational methods in nucleic acid structure modeling can be found in Louise-May et al. (1996) and in UNIT 7.5.

Many of the computational programs for calculating macromolecular structures are available on the internet. Secondary structure prediction programs for RNA (http://www. ibc.wustl.edu/ zuker/rnalform1.cgi) and DNA (http://sun2.science.wayne.edu/ -jslsun2/servers/dna/form 1.cgi) use experimentally determined thermodynamic parameters to find base-paired arrangements with the lowest and near-lowest free energies.

MC-SYM (http://www.iro.umontreal.ca/ $\sim$ major/mcsym.html) is a structural modeling program that uses published nucleic acid structures as a database to calculate three-dimensional structures from the sequence and experimental constraints. Standard double-helix geometry is used for the base-paired regions. A wide range of possible loops and bulges are modeled for the remaining regions based on published structures. Experimental data such as chemical reactivity of the nucleotides, cross-linking results, and incomplete NMR 
constraints are used to help the investigator choose between possible structures.

AMBER (http://www.amber.ucsf.edu/ amber/amber.html) and CHARMM (http:// yuri.harvard.edu/charmm/charmm.html) are programs that allow molecular dynamics simulations to be performed on nucleic acids and proteins. Coordinates and velocities are chosen at time zero for each atom in the macromolecule and solvent. After a time interval of $\sim 1$ fsec, potential energy functions are used to calculate the force, and thus the acceleration, on each atom in its new position. After each time step, new positions and new forces are calculated. The molecular motion of the macromolecule in solution can be simulated over a time scale of picoseconds to one nanosecond. The potential functions used to calculate the forces are clearly the key to a useful simulation. At present the potentials cannot fold an RNA into a correct structure without further information. However, if potentials derived from NMR measurements on the molecule (proton-proton distances and torsion angles) are added, an NMRderived structure is obtained. The quality of the structure will depend on the number and precision of the NMR restraints.

A goal to develop "Multiscale Modeling Tools for Structural Biology" is being supported by the NIH. The NIH Research Resource has a Web page (http://mmtsb.scripps.edu/) that has links to AMBER and CHARMM and also to Yammp, a molecular mechanics program for modeling structures such as ribosomes, viruses, and supercoiled DNA. Yammp uses a reduced representation, in which a sugar, base, or basepair is represented by a single "atom." Helices and proteins can be modeled by cylinders or spheres. The ultimate goal is to be able to model any molecular biological structure at any scale of representation needed to answer the questions of interest.

\section{SINGLE-MOLECULE METHODS}

Methods that measure the properties of single molecules can provide unique information. Measurements made in solution or in crystals are clearly the average properties of many molecules. With single-molecule detection, each different molecule can be studied in its own microenvironment. A review of the results from the early days of single-molecule research (1980s) has been published (Bustamante, 1991).

In principle, any type of spectroscopy can be applied to a single molecule, but the sensitivity required to do NMR spectroscopy has yet to be attained. A single electron magnetic mo- ment can be detected, but detection of a single proton magnetic moment requires about a 1000-fold increase in sensitivity (Rugar et al., 1994). Fluorescence and absorption spectroscopy have been measured on single molecules. Fluorescence energy transfer between two fluorophores depends on the distance $\left(1 / r^{6}\right)$ and angle between the fluorophores. It is common to assume random angular orientation between the fluorophores, which leads to an uncertainty in the distance measurement. If only two fluorophores are observed, either within one molecule or in two molecules, the absolute orientation of each fluorophore can be measured from the polarization of the fluorescence. This measurement determines the angular orientation and produces a more accurate distance (Ha et al., 1996). Spectroscopy of individual molecules has revealed the existence of longlived "dark" excited states (excited states that do not emit light) in addition to the previously known excited singlet and triplet states (Dickson et al., 1996, 1997).

Mechanical properties (such as elasticity) of single DNA molecules have been analyzed by measuring the force (piconewtons) necessary to stretch the molecule from a random coil to a stretched rod (Smith et al., 1992). Laser tweezers can be used to manipulate macromolecules in various ways; some recent applications have been illustrated on the muscle protein titin (Kellermayer et al., 1997; Rief et al., 1997).

Scanning-probe microscopy includes atomic force microscopy (AFM) and scanning tunneling microscopy (STM; Miles, 1997). A very fine tip is scanned across the sample attached to a surface, and the force (AFM) or the current (STM) is measured. The AFM method, which is the most useful for biological molecules, provides the height of the molecule as a function of the position of the scanning probe. Measurements can be made with the sample immersed in solution, allowing biochemical reactions to be followed. The resolution of the method (a few angstroms) allows imaging of duplex DNA and of the transcription of DNA by RNA polymerase (Kasas et al., 1997; Rippe et al., 1997). By coating the scanning tip with different surfaces, chemical forces between the tip and the sample molecule can be measured. Hydrogen bonding in nucleic acid bases (Boland and Ratner, 1995) and protein-ligand interactions (Chilkoti et al., 1995) have been measured in this manner.

Single-molecule methods are just being developed and applied. The ability to hold,
Biophysical Analysis of Nucleic Acids 
move, stretch, and observe individual nucleic acid and protein molecules allows, for the first time, directmeasurementof theforcesinvolved in biochemical reactions. The coupling of mechanical and chemical forces can be measured and controlled. Molecular motions can be directly observed, as demonstrated for the rotation of the ATP synthase enzyme (Noji et al., 1997).

\section{LITERATURE CITED}

Allain, F.T. and Varani, G. 1997. How accurately and precisely can RNA structure be determined by NMR? J. Mol. Biol. 267:338-351.

Baeyens, K.J., De Bondt, H.L., Pardi, A., and Holbrook, S.R. 1996. A curved RNA helix incorporating an internal loop with G.A and A.A nonWatson-Crick base pairing. Proc. Natl. Acad. Sci. U.S.A. 93:12851-12855.

Batey, R.T., Inada, M., Kujawinski, E., Puglisi, J.D., and Williamson, J.R. 1992. Preparation of isotopically labeled ribonucleotides for multidimensional NMR spectroscopy of RNA. Nucl. Acids Res. 20:4515-4523.

Battiste, J.L., Tan, R., Frankel, A.D., and Williamson, J.R. 1994. Binding of an HIV Rev peptide to Rev responsive element RNA induces formation of purine-purine base pairs. Biochemistry 33:2741-2747.

Biou, V., Yaremchuk, A., Tukalo, M., and Cusack, S. 1994. The $2.9 \AA$ crystal structure of T. thermophilus Seryl-tRNA synthetase complexed with tRNA $^{\text {Ser }}$. Science 263:1404-1410.

Boland, T. and Ratner, B.D. 1995. Direct measurement of hydrogen bonding in DNA nucleotide bases by atomic force microscopy. Proc. Natl. Acad. Sci. U.S.A. 92:5297-5301.

Bustamante, C. 1991. Direct observation and manipulation of single DNA molecules using fluorescence microscopy. Annu. Rev. Biophys. Biophys. Chem. 20:415-446.

Butcher, S.E., Dieckmann, T., and Feigon, J. 1997. Solution structure of a GAAA tetraloop receptor RNA. EMBO J. 16:7490-7499.

Cate, J.H. and Doudna, J.A. 1996. Metal binding sites in the major groove of a large ribozyme domain. Structure 4:1221-1230.

Cate, J.H., Gooding, A.R., Podell, E., Zhou, K., Golden, B.L., Kundrot, C.E., Cech, T.R., and Doudna, J.A. 1996a. Crystal structure of a group I ribozyme domain: Principles of RNA packing. Science 273:1678-1685.

Cate, J.H., Gooding, A.R., Podell, E., Zhou, K., Golden, B.L., Szewczak, A.A., Kundrot, C.E., Cech, T.R., and Doudna, J.A. 1996b. RNA tertiary structure mediation by adenosine platforms. Science 273:1696-1699.

Biophysical Analysis of Nucleic Acids
Cate, J.H., Hanna, R.L., and Doudna, J.A. 1997. A magnesium ion core at the heart of a ribozyme domain. Nature Struct. Biol. 4:553-558.
Chang, K.-Y. and Tinoco, I. Jr. 1997. The structure of an RNA "kissing" hairpin complex of the HIV TAR hairpin loop and its complement. J. Mol. Biol. 269:52-66.

Chen, X., Ramakrishnan, B., and Sundaralingam, M. 1997. Crystal structures of the side-by-side binding of distamycin to AT-containing DNA octamers d(ICITACIC) and d(ICATATIC). $J$. Mol. Biol. 267:1157-1170.

Cheong, C. and Moore, P.B. 1992. Solution structure of an unusually stable RNA tetraplex containing G- and U-quartet structures. Biochemistry 31:8406-8414.

Chilkoti, A., Boland, T., Ratner, B.D., and Stayton, P.S. 1995. The relationship between ligand-binding thermodynamics and protein-ligand interaction forces measured by atomic force microscopy. Biophys. J. 69:2125-2130.

Chou, S.H., Zhu, L., and Reid, B.R. 1997. Sheared purine-purine pairing in biology. J. Mol. Biol. 267:1055-1067.

Clegg, R.M., Murchie, A.I., Zechel, A., and Lilley, D.M. 1993. Observing the helical geometry of double-stranded DNA in solution by fluorescence resonance energy transfer. Proc. Natl. Acad. Sci. U.S.A. 90:2994-2998.

Correll, C.C., Freeborn, B., Moore, P.B., and Steitz, T.A. 1997. Metals, motifs, and recognition in the crystal structure of a 5S rRNA domain. Cell 91:705-712.

Dickson, R.M., Norris, D.J., Tzeng, Y.L., and Moerner, W.E. 1996. Three-dimensional imaging of single molecules solvated in pores of poly(acrylamide) gels. Science 274:966-969.

Dickson, R.M., Cubitt, A.B., Tsien, R.Y., and Moerner, W.E. 1997. On/off blinking and switching behaviour of single molecules of green fluorescent protein. Nature 388:355-358.

Dieckmann, T., Butcher, S.E., Sassanfar, M., Szostak, J.W., and Feigon, J. 1997. Mutant ATPbinding RNA aptamers reveal the structural basis for ligand binding. J. Mol. Biol. 273:467-478.

Doucet, J., Benoit, J.P., Cruse, W.B., Prange, T., and Kennard, O. 1989. Coexistence of A- and B-form DNA in a single crystal lattice. Nature 337:190192.

Fan, P., Suri, A.K., Fiala, R., Live, D., and Patel, D.J. 1996. Molecular recognition in the FMN-RNA aptamer complex. J. Mol. Biol. 258:480-500.

Farber, G.K. 1997. Laue crystallography: Lights! Camera! Action! Curr. Biol. 7:R352-R354.

Foldesi, A., Yamakage, S.I., Nilsson, F.P., Maltseva, T.V., and Chattopadhyaya, J. 1996. The use of non-uniform deuterium labelling ['NMR-window'] to study the NMR structure of a 21 mer RNA hairpin. Nucl. Acids Res. 24:1187-1194.

Fourmy, D., Recht, M.I., Blanchard, S.C., and Puglisi, J.D. 1996. Structure of the A site of Escherichia coli $16 \mathrm{~S}$ ribosomal RNA complexed with an aminoglycoside antibiotic. Science 274:1367-1371. 
Franklin, R.E. and Gosling, R.G. 1953. Molecular configuration in sodium thymonucleate. Nature 171:740-741.

Glusker, J.P., Lewis, M., and Rossi, M. 1994. Crystal Structure Analysis for Chemists and Biologists. VCH Publishers, New York.

Gray, D.M., Hung, S.-H., and Johnson, K.H. 1995. Absorption and circular dichroism spectroscopy of nucleic acid duplexes and triplexes. Methods Enzymol. 246:19-34.

Ha, T., Enderle, T., Ogletree, D.F., Chemla, D.S., Selvin, P.R., and Weiss, S. 1996. Probing the interaction between two single molecules: Fluorescence resonance energy transfer between a single donor and a single acceptor. Proc. Natl. Acad. Sci. U.S.A. 93:6264-6268.

Holbrook, S.R., Cheong, C., Tinoco, I. Jr., and Kim, S.-H. 1991. Crystal structure of an RNA double helix incorporating a track of non-Watson-Crick base pairs. Nature 353:579-581.

Special issue: James, T.L. (ed.). 1995. Nuclear magnetic resonance and nucleic acids. Methods Enzymol. 261:1-644.

Jiang, F., Kumar, R.A., Jones, R.A., and Patel, D.J. 1996. Structural basis of RNA folding and recognition in an AMP-RNA aptamer complex. $\mathrm{Na}$ ture 382:183-186.

Johnson, K.H. and Gray, D.M. 1992. Analysis of an RNA pseudoknot structure by CD spectroscopy. J. Biomol. Struct. Dyn. 9:733-745.

Kang, C.H., Zhang, X., Ratliff, R., Moyzis, R., and Rich, A. 1992. Crystal structure of four-stranded oxytricha telomeric DNA. Nature 356:126-131.

Kasas, S., Thomson, N.H., Smith, B.L., Hansma, H.G., Zhu, X., Guthold, M., Bustamante, C., Kool, E.T., Kashlev, M., and Hansma, P.K. 1997. Escherichia coli RNA polymerase activity observed using atomic force microscopy. Biochemistry 36:461-468.

Kellermayer, M.S., Smith, S.B., Granzier, H.L., and Bustamante, C. 1997. Folding-unfolding transitions in single titin molecules characterized with laser tweezers. Science 276:1112-1116.

Kieft, J.S. and Tinoco, I. Jr. 1997. Solution structure of a metal-binding site in the major groove of RNA complexed with cobalt (III) hexammine. Structure 5:713-721.

LeCuyer, K.A. and Crothers, D.M. 1994. Kinetics of an RNA conformational switch. Proc. Natl. Acad. Sci. U.S.A. 91:3373-3377.

Lipari, G. and Szabo, A. 1982. Model-free approach to the interpretation of nuclear magnetic resonance relaxation in macromolecules. J. Am. Chem. Soc. 104:4546-4559.

Lipscomb, L.A., Peek, M.E., Morningstar, M.L., Verghis, S.M., Miller, E.M., Rich, A., Essigmann, J.M., and Williams, L.D. 1995. X-ray structure of a DNA decamer containing 7,8-dihydro-8-oxoguanine. Proc. Natl. Acad. Sci. U.S.A. 92:719-723.
Louise-May, S., Auffinger, P., and Westhof, E. 1996. Calculations of nucleic acid conformations. Curr. Opin. Struct. Biol. 6:289-298.

Maglott, E.J. and Glick, G.D. 1997. A new method to monitor the rate of conformational transitions in RNA. Nucl. Acids Res. 25:3297-3301.

Miles, M. 1997. Scanning probe microscopy. Probing the future. Science 277:1845-1847.

Murchie, A.I.H., Clegg, R.M., von Kitzing, E., Duckett, D.R., Diekmann, S., and Lilley, D.M.J. 1989. Fluorescence energy transfer shows that the fourway DNA junction is a right-handed cross of antiparallel molecules. Nature 341:763-766.

Nikonowicz, E.P., Sirr, A., Legault, P., Jucker, F.M., Baer, L.M., and Pardi, A. 1992. Preparation of ${ }^{13} \mathrm{C}$ and ${ }^{15} \mathrm{~N}$ labelled RNAs for heteronuclear multi-dimensional NMR studies. Nucl. Acids Res. 20:4507-4513.

Noji, H., Yasuda, R., Yoshida, M., and Kinosita, K. Jr. 1997. Direct observation of the rotation of F1-ATPase. Nature 386:299-302.

Passner, J.M. and Steitz, T.A. 1997. The structure of a CAP-DNA complex having two cAMP molecules bound to each monomer. Proc. Natl. Acad. Sci. U.S.A. 94:2843-2847.

Pease, A.C., Solas, D., Sullivan, E.J., Cronin, M.T., Holmes, C.P., and Fodor, S.P. 1994. Light-generated oligonucleotide arrays for rapid DNA sequence analysis. Proc. Natl. Acad. Sci. U.S.A. 91:5022-5026.

Pley, H.W., Flaherty, K.M., and McKay, D.B. 1994. Three-dimensional structure of a hammerhead ribozyme. Nature 372:68-74.

Puglisi, J.D., Chen, L., Blanchard, S., and Frankel, A.D. 1995. Solution structure of a bovine immunodeficiency virus Tat-TAR peptide-RNA complex. Science 270:1200-1203.

Radhakrishnan, I. and Patel, D.J. 1994. Solution structure and hydration patterns of a pyrimidine.purine-pyrimidine DNA triplex containing a novel T.CG base-triple. J. Mol. Biol. 241:600619.

Ramos, A., Gubser, C.C., and Varani, G. 1997. Recent solution structures of RNA and its complexes with drugs, peptides and proteins. Curr. Opin. Struct. Biol. 7:317-323.

Recht, M.I., Fourmy, D., Blanchard, S.C., Dahlquist, K.D., and Puglisi, J.D. 1996. RNA sequence determinants for aminoglycoside binding to an A-site rRNA model oligonucleotide. $J$. Mol. Biol. 262:421-436.

Rief, M., Gautel, M., Oesterhelt, F., Fernandez, J.M., and Gaub, H.E. 1997. Reversible unfolding of individual titin immunoglobulin domains by AFM. Science 276:1109-1112.

Rippe, K., Guthold, M., von Hippel, P.H., and Bustamante, C. 1997. Transcriptional activation via DNA-looping: Visualization of intermediates in the activation pathway of $E$. coli RNA polymerase $\mathrm{x}$ sigma 54 holoenzyme by scanning force microscopy. J. Mol. Biol. 270:125-138.
Biophysical Analysis of Nucleic Acids 
Roberts, G.C.K. 1993. NMR of Macromolecules. A Practical Approach. IRL Press, New York.

Rugar, D., Zuger, O., Hoen, S., Yanonni, C.S., Vieth, H.-M., and Kendrick, R.D. 1994. Force detection of nuclear magnetic resonance. Science 264:1560-1563.

Saenger, W. 1984. Principles of Nucleic Acid Structure. Springer-Verlag, New York.

SantaLucia, J. Jr., Shen, L.X., Cai, Z., Lewis, H., and Tinoco, I. Jr. 1995. Synthesis and NMR of RNA with selective isotopic enrichment in the base moieties. Nucl. Acids Res. 23:4913-4921.

Scott, W.G., Finch, J.T., and Klug, A. 1995. The crystal structure of an all-RNA hammerhead ribozyme: A proposed mechanism for the RNA catalytic cleavage. Cell 81:991-1002.

Scott, W.G., Murray, J.B., Arnold, J.R.P., Stoddard, B.L., and Klug, A. 1996. Capturing the structure of a catalytic RNA intermediate: The hammerhead ribozyme. Science 274:2065-2069.

Shen, L.X. and Tinoco, I. Jr. 1995. The structure of an RNA pseudoknot that causes efficient frameshifting in mouse mammary tumor virus. $J$. Mol. Biol. 247:963-978.

Smith, S.B., Finzi, L., and Bustamante, C. 1992. Direct mechanical measurements of the elasticity of single DNA molecules by using magnetic beads. Science 258:1122-1126.

Tinoco, I. Jr. and Kieft, J.S. 1997. The ion core in RNA folding. Nature Struct. Biol. 4:509-512.

Tolbert, T.J. and Williamson, J.R. 1996. Preparation of specifically deuterated RNA for NMR studies using a combination of chemical and enzymatic synthesis. J. Am. Chem. Soc. 118:7929-7940.

Tuschl, T., Gohlke, C., Jovin, T.M., Westhof, E., and Eckstein, F. 1994. A three-dimensional model for the hammerhead ribozyme based on fluorescence measurements. Science 266:785-789.

Varani, G. 1997. RNA-protein intermolecular recognition. Acc. Chem. Res. 30:189-195.
Varani, G. and Tinoco, I. Jr. 1991. RNA structure and NMR spectroscopy. Q. Rev. Biophys. 24:479-532.

Wang, E., Malek, S., and Feigon, J. 1992. Structure of a G.T.A triplet in an intramolecular DNA triplex. Biochemistry 31:4838-4846.

Watson, J.D. and Crick, F.H.C. 1953. Molecular structure of nucleic acids. Nature 171:737-738.

Wemmer, D. 2000. Structure and dynamics by NMR. In Nucleic Acids: Structures, Properties, and Functions (V.A. Bloomfield, D.M. Crothers, and I. Tinoco, Jr., eds.) pp. 111-163. University Science Books, Mill Valley, Calif.

Wemmer, D. and Dervan, P. 1997. Targeting the minor groove of DNA. Curr. Opin. Struct. Biol. 7:355-361.

Wilkins, M.H.F., Stokes, A.R., and Wilson, H.R. 1953. Molecular structure of deoxypentose nucleic acids. Nature 171:738-740.

Williamson, J.R. 1994. G-quartet structures in telomeric DNA. Annu. Rev. Biophys. Biomol. Struct. 23:703-786.

Wu, M. and Turner, D.H. 1996. Solution structure of (rGCGGACGC) 2 by two-dimensional NMR and the iterative relaxation matrix approach. Biochemistry 35:9677-9689.

Wu, M., McDowell, J.A., and Turner, D.H. 1995. A periodic table of symmetric tandem mismatches in RNA. Biochemistry 34:3204-3211.

Wüthrich, K. 1986. NMR of Proteins and Nucleic Acids. John Wiley \& Sons, New York.

Zimmerman, G.R., Jenison, R.D., Wick, C.L., Simorre, J.-P., and Pardi, A. 1997. Interlocking structural motifs mediate discrimination by a theophylline-binding motif. Nature Struct. Biol. 4:644-648.

Contributed by Ignacio Tinoco, Jr.

University of California

Lawrence Berkeley National Laboratory

Berkeley, California 